\title{
OPTICAL IMAGERY OF NOVA REMNANTS
}

\author{
Richard A. Wade \\ Steward Observatory, University of Arizona, Tucson, Arizona 85721
}

\begin{abstract}
Resolved nebular remnants of classical novae are discussed in terms of size, surface brightness, and shape. A list of 26 known resolved remnants is given. The conditions necessary for visibility of a resolved remnant are discussed. The structure of remnants can be studied at optical wavelengths by direct imaging and by long-slit or otherwise spatially resolved spectroscopy of emission lines. Based on recent spatiokinematic studies, most nebular remnants are prolate in outline, with sub-structure that has been characterized as consisting of "polar blobs" and "equatorial rings". The mechanism that shapes the remnants is not yet known with certainty. There is room for much additional work in discovering new remnants and in characterizing those that are known.
\end{abstract}

\section{Preliminaries}

This review is about the occurrence, size, shape, and brightness of resolved nebular remnants of classical novae. The physical conditions in the nebular remnants are not discussed. The literature review on which this paper is based was concluded in June 1989. That review was aided greatly by H. W. Duerbeck's (1987a) monograph, $A$ Reference Catalogue and Atlas of Galactic Novae, and by SIMBAD, a database of the Strasbourg (France) Astronomical Data Center.

It is difficult to find a term that refers unambiguously and at the same time briefly to the subject of this paper. Envelope is too easily confused with the same term in stellar interiors work. Ejecta could refer to material observed at any stage of the nova outburst, whether or not it is resolved, or even to material escaping via a wind from a red giant. The word shell, used without the qualifying word nova, could connote something to do with Be stars. In Astronomy and Astrophysics Abstracts one can find references to angularly resolved gas surrounding a stellar nova remnant under any of these headings. Even the phrase nebular remnant is ambiguous, because while it ought to suggest a cloudy or diffuse appearance and hence something that has perceptible angular or spatial extent, it can also refer to unresolved gas in the "nebular" phase of the nova outburst, which is defined spectroscopically by the presence of "nebular" emission lines. It seems that the clumsy phrase resolved nebular remnant is required in 
order for all confusion to be dispelled, but relying on context it is probably sufficient to write or say nebular remnant or nova shell.

\section{Occurrence of Nebular Remnants of Novae}

Presumably every classical nova explosion ejects a cloud of material into circumstellar space. For various reasons discussed below, these clouds are not always observed. Excluding $\eta \mathrm{Car}$, the number of nebular remnants that are known or claimed is 26 . References to discussions in the literature for most of these can be found in Duerbeck (1981, 1987a), while additional detections (some of which are admittedly marginal) are discussed by Cohen $(1985,1988)$. Additional up-to-date references can be located through SIMBAD. These 26 remnants are associated with the following novae:

I. References in Duerbeck (1987a, except for FH Ser). V603 Aql, V605 Aql, T Aur, T Crb, V476 Cyg, V1500 Cyg, HR Del, DQ Her, BT Mon, RS Oph (radio shell only), GK Per, RR Pic, CP Pup, T Pyx, FH Ser (Seitter and Duerbeck 1986), CK Vul.

II. From Cohen (1985, except for DN Gem). V500 Aql, V1229 Aql, DN Gem (Cohen 1988), V446 Her, V533 Her, DK Lac, XX Tau, RW UMi, LV Vul.

III. A nebular disk around Nova CP Lac was apparently observed by W. Baade (reported by Adams 1944 and McLaughlin 1945), but was not detected in the much later survey by Cohen and Rosenthal (1983). Since the inferred rate of angular expansion has been used frequently in the literature (e.g. McLaughlin 1960 and Cohen 1985), I include this object for completeness and hope that someday Baade's plates will be published.

Published images regrettably do not seem to exist for all of these resolved remnants, and not all of those that do exist are adequately labelled, e.g. with orientation and scale of the picture. Many images can be traced through the references given by Duerbeck (1987a). Some additional recent images can be found in Barden and Wade (1988: DQ Her), Cohen (1985: V500 Aql, V1500 Cyg, V533 Her, XX Tau, LV Vul, CK Vul), Duerbeck (1987b: V603 Aql, V476 Cyg), Duerbeck (1987c: BT Mon, RR Pic, CP Pup, T Pyx), Seitter and Duerbeck (1986: FH Ser, GK Per), Shara et al. (1989: T Pyx), and some contributions to this conference, e.g. Wade et al. (1990: V1500 Cyg) and the conference poster.

Cohen (1985) has looked for resolved nebular remnants around the following novae, without finding them: IV Cep, Q Cyg, V450 Cyg, DI Lac, HR Lyr, V841 Oph, WY Sge, V368 Sct, V373 Sct. Menzies, O'Donoghue, and Warner (1986) found no $\mathrm{H} \alpha$ emission around the peculiar dwarf nova BV Cen, which they propose may have been a nova similar to GK Per. Ellis, Grayson, and Bond (1984) noted diffuse cmission around the cataclysmic variable $0623+71$ and suggested that it might be a nebular remnant from a nova outburst. 
Cohen (1988) has discussed the observability of resolved remnants in terms of the extinction-corrected apparent magnitude at maximum brightness $m_{V}-A_{V}$, the expansion velocity of the gas, and the time since outburst. Cohen's empirical rule essentially states that a nova shell will be simultaneously resolvable and detectable if $m_{V}-A_{V}<7.5$ and not too great a time interval has elapsed since outburst. On this basis, she expects to detect 10-12 additional resolved nebular remnants within a few years, using the same observing technique described in Cohen and Rosenthal (1983) and Cohen (1985). (Cohen has restricted her attention to novae that were well-observed at maximum brightness, since her interest is in refining the maximum-magnitude-rate-of-decline, or MMRD, relation. For a study of the physics of shell ejection or of the shells themselves, this restriction is unnecessary and additional recent novae may profitably be studied. The BT Mon remnant falls into this category, see Marsh, Wade, and Oke 1983, Schaefer and Patterson 1983, and Duerbeck 1987c.)

Cohen's criterion for resolvability is suitable for ground-based searches where no attempt at image sharpening is made. A more general rule would need to take into account the possibility of using speckle interferometry or image sharpening (by active optics or in analysis software). The angle between the stellar nova remnant and a parcel of gas expelled at the "moment" of outburst is given by

$$
\theta=(0.207 \operatorname{arcsec}) \times\left(\frac{v \Delta T}{d}\right)
$$

where $v$ is the speed of the gas in $\mathrm{km} \mathrm{sec}^{-1}$ tangential to the line of sight, $\Delta T$ is the time since outburst in years, and $d$ is the distance of the nova in pc. Thus if $v=1000 \mathrm{~km} \mathrm{sec}-1$ and $d=1 \mathrm{kpc}$, the remnant should have a radius of about 1 arcsec suitable for ground-based detection in 5 years. If the required angular size is only $\sim 0.2$ arcsec as appropriate to the Hubble Space Telescope (HST), the delay after outburst is reduced, or shells with smaller expansion velocities or that are further away can be resolved within a reasonable time. For example, imaging with HST could resolve a nebular remnant at $10 \mathrm{kpc}$ after 19 years, even if $v=500 \mathrm{~km} \mathrm{sec}{ }^{-1}$. Finally, if enough photons were available, speckle interferometric techniques could resolve (radius $=0.01$ arcsec $)$ a nova shell at the distance of the LMC $(50 \mathrm{kpc})$ after only 2.4 years, for $v=1000 \mathrm{~km} \mathrm{sec}^{-1}$. Speckle imaging seems so far to have been attempted only for Nova V1500 Cyg (1975), see Blazit et al. (1977); of course, SN1987A has been the object of considerable speckle interferometric work.

The second part of Cohen's rule refers to the strong time-dependence of the surface brightness of the nebular remnant. The angular radius $r$ of the remnant is proportional to $v \Delta T$, while the emission measure of the gas varies according to

$$
\text { E.M. } \propto n_{e}^{2} V \propto(v \Delta T)^{-3}
$$

where $V$ is the volume occupied by the gas. The nebula is here assumed to be matterbounded. The surface brightness $\Sigma$ of the nebula therefore varies as

$$
\Sigma \propto \text { E.M. } \times r^{-2} \propto(v \Delta T)^{-5} .
$$


Whether a nova shell can successfully be detected is therefore a matter of how rapidly it expands compared with how rapidly is fades from view.

For the sake of concreteness, consider the expected photon rate in the $\mathrm{H} \alpha$ emission line from a resolved nebular remnant. Assume Case A recombination at a kinetic temperature of a few $\times 10^{3} \mathrm{~K}$. Then the photon rate per unit solid angle can be expressed approximately by

$$
\begin{aligned}
\Sigma(\mathrm{H} \alpha)= & \left(1.3 \times 10^{3} \mathrm{~cm}^{-2} \mathrm{~s}^{-1} \operatorname{arcsec}^{-2}\right) \times\left(\frac{M_{\text {neb }}}{10^{-4} M_{\odot}}\right)^{2} \times \\
& \left(\frac{v}{1000 \mathrm{~km} \mathrm{sec}^{-1}}\right)^{-5} \times\left(\frac{\Delta T}{1 \text { year }}\right)^{-5} \times\left(\frac{\epsilon \zeta_{1}}{f \zeta_{2}^{2}}\right)
\end{aligned}
$$

where $M_{\text {neb }}$ is the mass of gas in the nebular remnant, $f$ is the geometrical filling factor, $\varsigma_{1}=n_{p} / n_{e}$ and $\varsigma_{2}=M_{n e b} /\left(f V\left\langle n_{e}\right\rangle m_{p}\right)$ are factors that depend on the chemical composition and ionization state of the gas, and $\epsilon=\left\langle n_{e}^{2}\right\rangle /\left\langle n_{e}\right\rangle^{2}$ is a clumping factor. Here $V$ is the "enveloping volume" of the nebula, and $f$ and $\epsilon$ take into account inhomogeneities on large and small size scales, respectively. Note that $f\langle 1$ and $\epsilon>1$. If the nebula is hydrogen rich (!) and close to being fully ionized, then $\zeta_{1}$ and $\zeta_{2}$ are not far from unity. The expression assumes that the nebula is resolved, and it ignores interstellar extinction. Aside from extinction, the surface brightness of a resolved source does not depend upon the distance of the nova.

To see what this means in practice, consider a nova shell that has been expanding at $1000 \mathrm{~km} \mathrm{sec}^{-1}$ for 50 years. Assume $M_{\text {neb }}=10^{-4} M_{\odot}$ and set $f=\epsilon=\varsigma_{1}=$ $\varsigma_{2}=1$ for simplicity. A 1 -hour exposure using a $60 \%$ quantum efficient CCD at a $4-\mathrm{m}$ telescope would detect about 120 photons per 0.6 -arcsec square pixel from this object. (Reasonable estimates of telescope and filter efficiencies and atmospheric extinction have been factored in.) If $d=1 \mathrm{kpc}$, the radius of the nebula would be $10 \mathrm{arcsec}$, and the nebula would project onto of order 1000 pixels. It is an open question whether a 50-year old nova shell would be fully ionized and still warm, but this naive calculation shows that it is not out of the question to discover additional shells of modest age and angular size.

\section{Expansion Distances}

Distances of novae derived from comparing the angular expansion rate of the nebular remnant with the line-of-sight velocity of the emitting gas have been discussed at length by Ford and Ciardullo (1988) and Cohen (1988) and more briefly by others at the Victoria symposium on the extragalactic distance scale (van den Bergh and Pritchet 1988). The article by Cohen provides a significant update to her older discussion of nova shells (Cohen 1985). Novae have been used as tertiary distance indicators for extragalactic objects, via the MMRD relation, whose calibration rests in part on expansion distances. 
Deriving an expansion distance is straightforward in principle, but there are several difficulties that can lead to systematic errors. Deceleration of the expanding gas if it interacts with the interstellar medium (or a pre-existing circumstellar cloud), or acceleration of the gas by a subsequent fast wind from the stellar nova remnant, will lead to an erroneous estimate of the angular expansion rate (Duerbeck 1987b). Comparing angular sizes of a nebular remnant from images obtained at early and late epochs may lead to a false inferrence that deceleration is present and an erroneous correction for it, when the early image of the remnant is similar in size to the seeing disk (cf. the discussion of DQ Her by Ferland 1980).

The most serious difficulties encountered in deriving expansion distances involve assigning a tangential speed to the gas at the projected edge of the resolved nebula. This speed is not directly observable and is adopted based on an observed line-of-sight speed of the gas. This line-of-sight measurement, however, is often from observations early in the outburst, sometimes from an absorption spectrum, rather than measured from the emission spectrum of the nebula itself.

\section{Shapes of Nova Shells}

The line-of-sight speed must be converted to a tangential speed with the use of a model for the geometry of the nebula. The simplest assumption is that the nebula is expanding spherically, so that these two speeds are the same. The shapes of wellresolved nova shells, however, are observed not to be circular in outline. The next level of approximation is to assume that the nebula is an ellipsoid of revolution, either prolate or oblate, and to infer somehow the axis ratio and the orientation of this figure in three dimensions; the correct scaling of line-of-sight to tangential speeds follows from this. Ford and Ciardullo (1988) have discussed this problem in detail and find that errors approaching or even exceeding $20 \%$ can be introduced systematically into the MMRD relation if an incorrect geometrical model is used.

Evidence on the shape of a nova shell comes directly from (1), direct imaging of the shell in the light of emission lines, or from (2), detailed spectroscopic study of the resolved nebular remnant, using long-slit or other techniques to sample many different lines of sight through the shell and interpreting the results with a so-called spatiokinematic model. Indirect evidence on shapes comes from (3), interpreting the emission line profiles from the unresolved (early) nebular remnant.

Method (1) deals with a projected image of the shell only, but in favorable cases this image betrays a basic symmetry pattern that may lead to an improved geometrical description of the nebula. The most famous example is of course the nebula surrounding Nova DQ Her, but it is important to note that, based on images alone, it is still difficult to decide whether this shell is oblate or prolate.

Method (2) can be followed using small or large amounts of data. The case of DQ Her again illustrates this nicely. Ford and Ciardullo (1988) discuss a prolate ellipsoidal 
model based on one long-slit spectrum and symmetries assumed from direct imaging. (The Jensen photograph of the DQ Her remnant in 1973 is reproduced much more clearly in Weaver 1974.) Cohen (1988) discusses a similar model supported by many long-slit spectra. Barden, Rabin, and Wade (1988, see also Barden and Wade 1988) obtained spectra at many overlapping positions in the nebula using an optical fiber array. In this last study, the spatial sampling was dense enough that is was possible to let the data "speak" almost directly concerning the shape of the nebula without the intervention of a smooth, regular model, but it is still true that an implicit symmetry was assumed to establish the relative scaling of the velocity (wavelength) and spatial (RA and Dec) axes. (It was found much easier to visualize the large amount of data gathered in this study by means of a movie that allows the viewer to "see" the nebula from a variety of aspect angles. The spatial patterns of the $\mathrm{H} \alpha$ and the $\mathrm{N}$ [II] emission are strikingly different.)

Method (3), exemplified by the study of three novae by Hutchings (1972), interprets intensity peaks and troughs in emission line profiles in terms of "polar blobs" and "equatorial rings", again with an implied assumption of azimuthal symmetry, if not an assumption that the overall figure (or rather its outline) is ellipsoidal.

Azimuthal symmetry about the orbital axis of the nova binary star system is indeed a plausible assumption, since the mass loss that feeds the nebula is understood to extend over a time interval long compared to the orbital period. Opinions have, however, varied over the years as to whether the nebula (always taken to be a thin shell or parts of a thin shell) has one long axis and two short, or vice versa. For example, Hutchings (1972) and Soderblom (1976) worked with an oblate model for the HR Del remnant. A prolate geometry, however, has generally been favored in recent years, as more kinematic studies of resolved remnants have been carried out. Examples are Solf's (1983) study of the HR Del remnant, Duerbeck and Seitter's (1987) study of GK Per, and the studies of DQ Her cited above. Nevertheless, without a convincing theoretical explanation for why remnants should be prolate, it is perhaps dangerous to assume this is generally true.

Many explanations for the overall shapes of nova shells have been offered. These include the effects of white dwarf rotation and pulsation (Warner 1972) or rotation alone (Fiedler and Jones 1980), magnetic fields (Mustel 1958), interactions of the expanding white dwarf envelope with a surrounding accretion disk (A. Shankar et al., this conference) or the secondary star (Sparks and Starrfield 1973), and a fast polar-directed wind after the nova outburst that shapes the nebula (Duerbeck $1987 \mathrm{c}$ ). Some additional schemes are catalogued by Martin (1989). Some of these models were put forward to explain or predict an oblate shape; now, of course, we think that the shells are prolate.

Quite apart from the overall, or outline, shape of nova shells is the question of their structure on smaller scales. One can identify what Professor Seitter has termed "meso-structure", such as the blobs and rings that are known from a few well-resolved nebular remnants and inferred from the emission line profiles of other novae. As already noted, the fine example of the DQ Her remnant shows that it is not just that the line emission is present at some "latitudes" and not others, but that the latitude distribution 
of emissivity varies from one species to another. Questions arise as to how universal this "banded" appearance is, how regular it is in any one remnant, and of course how this regularity comes about. In view of the very different estimates of nebular mass that come from radio studies on the one hand (Seaquist et al. 1980; R. Hjellming, this conference) and optical studies on the other, one must also confront the question of whether these variations of line surface brightness with position are due to variations in mass density, or whether temperature, ionization, or composition differences are the cause. Is the optical line emission an accurate tracer of the ejected material?

Fine structure, in the sense of several closely spaced velocity components observed in an emission line at a particular location, has been observed in the HR Del remnant (Gallagher and Anderson 1976) and recently in the DQ Her remnant (S.C. Barden and S.C. Wolf, unpublished echelle observations). It would be good to know better at what point in the nova outburst or decline this fine structure arises, and how common it is, but the observations are difficult. Corresponding to this fine velocity structure, one expects to see brightness or line ratio variations at small angular scales. Possibly HST will provide such observations.

\section{Desiderata}

There are few branches of astrophysics where the old refrain, "More observations are needed!", is more applicable than to the study of resolved nebular remnants of classical novae. By the time a particular remnant has reached a size large enough to be resolved, it is also fading very rapidly. Therefore most of what we know about the structure of nova shells comes from observations of a handful of relatively nearby objects, which may not even be typical. Even very basic data about more shells is welcome information.

Observers should bear in mind that timing is important. It pays to keep trying to resolve the nebular remnants of recent novae. Negative results have their uses and should be reported. It is also important to report as much quantitative information as possible: size, shape, flux, surface brightness and contrast. Modern image processing techniques should be exploited whenever possible to remove the bright central star and to sharpen the point spread function of the image. When new resolved nova shells are found, spatially resolved spectroscopy should be carried out, with a view to testing the models of blobs and rings and determining case by case whether the remnants are essentially prolate or oblate. We always learn more from the exceptional cases.

\section{Concluding Remarks}

Three decades ago, Cecilia Payne-Gaposchkin (1958) was able to report nebular expansion "parallaxes" (this term is now obsolete) for seven classical novae (V603 Aql, V476 Cyg, CP Lac, GK Per, RR Pic, DQ Her, and T Aur). We now have a sample of resolved nebulae that is three times larger. Unfortunately they are not uniformly well-studied. This is partly the result of the nature of the problem: nova shells are 
faint and small, and they fade rapidly. But it is also an indication that more effort can profitably be applied.

I acknowledge helpful discussions with my collaborators, S. Barden, R. Ciardullo, G. Jacoby, and D. Rabin. Preparation of this review was supported by National Science Foundation grant AST-8818069 to the University of Arizona.

\section{References}

Adams, W.S. 1944, Pub. A.S.P., 56, 218.

Barden, S.C., Rabin, D.M., and Wade, R.A. 1988, Bull. AAS, 20, 1052.

Barden, S.C., and Wade, R.A. 1988, in A.S.P. Conference Series S, Fiber Optics in Astronomy, ed. S.C. Barden (San Francisco: Astron. Soc. Pacific), p. 113.

Blazit, A., Bonneau, D., Koechlin, L., and Labeyrie, A. 1977, Ap. J. (Letters), 214, L79.

Cohen, J.G. 1985, Ap. J., 292, 90.

- 1988, in A.S.P. Conference Series 4, The Extragalactic Distance Scale, ed. S. van den Bergh and C. J. Pritchet (San Francisco: Astron. Soc. Pacific), p. 114.

Cohen, J.G., and Rosenthal, A.J. 1983, Ap. J., 268, 689.

Duerbeck, H.W. 1981, Pub. A.S.P., 93, 165.

- 1987a, A Reference Catalogue and Atlas of Galactic Novae (Dordrecht: D. Reidel), reprinted from Space Science Rev., 45, 1.

- 1987b, Astr. Sp. Sci., 131, 461.

—. 1987 c, ESO Messenger, Nr. 50, 8.

Ellis, G.L., Grayson, E.T., and Bond, H.E. 1984, Pub. A.S.P., 96, 283.

Duerbeck, H.W., and Seitter, W.C. 1987, Astr. Sp. Sci., 131, 467.

Ferland, G.J. 1980, Observatory, 100, 166.

Fiedler, R.L., and Jones, T.W. 1980, Ap. J., 239, 253.

Ford, H.C., and Ciardullo, R. 1988, in A.S.P. Conference Series 4, The Extragalactic Distance Scale, ed. S. van den Bergh and C. J. Pritchet (San Francisco: Astron. Soc. Pacific), p. 128.

Gallagher, J.S., and Anderson, C.M. 1976, Ap. J., 203, 625.

Hutchings, J.B. 1972, M.N.R.A.S., 158, 177.

Marsh, T.R., Wade, R.A., and Oke, J.B. 1983, M.N.R.A.S., 250, 33P.

Martin, P.G. 1989, in Classical Novae, ed. M.F. Bode and A. Evans (Chichester: John Wiley and Sons), chapter 5.

McLaughlin, D.B. 1945, Pub. A.S.P., 57, 69.

-. 1960, in Stellar Atmospheres, ed. J.L. Greenstein (Chicago: University of Chicago Press), p. 585.

Menzies, J.W., O'Donoghue, D., and Warner, B. 1986, Astr. Sp. Sci., 122, 73.

Mustel, E.R. 1958, in IAU Symposium 6, Electromagnetic Phenomena in Cosmical Physics, ed. B. Lehnert (Cambridge: Cambridge University Press), 193.

Payne-Gaposchkin, C. 1958, in Handbuch der Physik LI, ed. S. Flügge (Berlin: SpringerVerlag), p. 752. 
Schaefer, B.E., and Patterson, J. 1983, Ap. J., 268, 710.

Seaquist, E.R., Duric, N., Israel, F.P., Spoelstra, T.A.T., Ulich, B.L., and Gregory, P.C. 1980, A. J., 85, 283.

Seitter, W.C., and Duerbeck, H.W. 1986, in RS Ophiuchi and the Recurrent Nova Phenomenon, ed. M.F. Bode (Utrecht: VNU Science Press), p. 71.

Shara, M.M., Moffat, A.F.J., Williams, R.E., and Cohen, J.G. 1989, Ap. J., 337, 720.

Soderblom, D. 1976, Pub. A.S.P., 88, 517.

Solf, J. 1983, $A p$. J., 273, 647.

Sparks, W.M., and Starrfield, S. 1973, M.N.R.A.S., 164, 1P.

van den Bergh, S., and Pritchet, C.J. 1988, eds., A.S.P. Conference Series 4, The Extragalactic Distance Scale (San Francisco: Astron. Soc. Pacific).

Wade, R.A., Ciardullo, R., DeVeny, J.B., Jacoby, G.H., and Schoening, W.E. 1990, in IAU Colloquium 122, Physics of Classical Novae, in press.

Warner, B. 1972, M.N.R.A.S., 160, 35P.

Weaver, H. 1974, in Highlights of Astronomy 3, ed. G. Contopolous (Dordrecht: D. Reidel), p. 509. 